Advanced tunnelling

\author{
W.A. Phillips
}

Principles of Electron Tunneling Spectroscopy. By E.L. Wolf. Clarendon: 1985. Pp.576. £60, $\$ 80$.

THE passage of particles through potential barriers, forbidden by classical physics, is convincing evidence in favour of quantum mechanics. Naturally occurring examples of this electron tunnelling are common. but the fabrication of sufficiently thin $(\sim 2 \mathrm{~nm})$ and perfect artificial barriers is not easy. The first examples of such barriers, apart from tunnel diodes, were aluminium-aluminium oxide--metal junctions (1959). Measurements of current as a function of voltage across the junction not only illustrated the phenomenon of tunnelling but also made an immediate impact when used to explore fundamental aspects of superconductivity. Most of the papers published on tunnelling in the following 15 years were concerned with superconductivity, and were very important in confirming the microscopic theory. Most dramatic of all was the discovery of Josephson tunnelling, where a current can pass from one superconductor to another through an insulating barrier even in the absence of a voltage across the junction, work which culminated in the award of a Nobel prize to Esaki, Giaever and Josephson in 1973.

More recently, the emphasis of research in this area has shifted and broadened. Inelastic tunnelling processes, where electrons lose energy as they tunnel, provide an additional channel for conduction above a threshold bias voltage which is equal to the characteristic energy of, for example, the vibrations of a molecule absorbed in the barrier. This novel spectroscopy, which needs only a voltmeter, is used to study the surface chemistry of absorbed molecules, vibrational states and other excitations in the barrier region. Of particular interest is a related process in which visible light is emitted indirectly by a tunnelling electron in a junction. Technically it is now possible to demonstrate tunnelling through a vacuum gap between a metal tip and a metal surface and so, because of the exponential variation of current with separation, to study surface topography in great detail. The preparation of sufficiently thin films on an atomic scale in the semiconductor industry suggests that interest in tunnelling effects and devices is going to increase sharply.

The appearance of a comprehensive account of electron tunnelling is therefore timely. Wolf has had to make a difficult choice between giving due weight to all aspects of the existing literature, or preparing the ground for future developments by under-playing mature fields of study. He has adopted the former approach, taking an encyclopaedic view

and including 250 figures and 1.250 references, mainly to the original literature. This has meant, inevitably, that roughly half of the book is devoted to aspects of tunnelling that involve superconductivity.

The other problem that Wolf has faced is that while the basic idea of electron tunnelling is relatively straightforward, many of the areas of physics where it can usefully be applied are not. For example, superconductivity, scattering by magnetic impurities or electron scattering by molecules are all complicated topics which have a much broader significance than he has been able to discuss. The consequent unevenness in depth of treatment would be a serious flaw in an introductory book in which complete coverage and continui-

\section{Ways of resistance}

\section{Peter I. Trigg}

Essential Malariology, 2nd Edn. By Leonard Jan Bruce-Chwatt. Heinemann Medical/Wiley:1985. Pp.452. Hbk £22.50, \$40. ELBS edition for sale in Africa, Asia and the West Indies, pbk£7.50.

MAlARIA is still the most important parasitic disease in the tropics. Excluding Africa south of the Sahara, 5.5 million cases were provisionally reported to the World Health Organization (WHO) in 1983 but the true incidence is estimated to be around 20 million cases per year. A further $\mathbf{2 0 0}$ million people in Africa are believed to be chronically infected, onethird of whom suffer acute manifestations.

This overall epidemiological picture has remained unchanged since the publication of the first edition of Essential Malariology in 1980. However, the technical constraints on malaria control, amply described by Professor Bruce-Chwatt, have increased. Probably the most important of them has been the spread of multiresistant Plasmodium falciparum in SouthEast Asia and Oceania. Chloroquine resistance has appeared in Africa and by 1984 had been reported in 15 countries of Central and East Africa. This situation has provoked a renaissance in malaria research, with, over the past five years, remarkable progress in the development and field testing of new antimalarial drugs, the development of prospective vaccines and the understanding of the epidemiology of the disease and its socioeconomic impact.

Against this backdrop, the new edition of Essential Malariology comes at an opportune time. Although it is a pity that it appeared too late to include all of WHO's recent recommendations on the use of antimalarial drugs - an understandable omission in such a rapidly moving field - it certainly provides, at a critical juncture in malaria control, the scientific background to recent parasitological, ty are essential, but matters much less in an advanced text such as this: a great deal of background knowledge is assumed and Wolf essentially summarizes the relevant literature on each aspect of the subject and points the reader towards the links between them.

Principles of Electron Tunneling Spectroscopy stands as an important guide to the experimental and theoretical advances of the past 25 years, giving due weight to established knowledge and hinting at developments to come. It will be used extensively by those working in the field.

W.A. Phillips is a Lecturer in Physics at the Cavendish Laboratory, University of Cambridge, Madingley Road, Cambridge CB3OHE, UK.

entomological, epidemiological and immunological research on human malaria and its transmission. In doing so it goes beyond the scope of the first edition. Professor Bruce-Chwatt faced a difficult problem in selecting practical information from the avalanche of recent publications, but he has admirably outlined important areas and has highlighted the relationship between basic research and its application.

The new edition also stresses practical methods of prevention, diagnosis, treatment and control in the context of the current policy of integrating malaria control into an overall primary health care approach. Various patterns of such integration have been developed in different countries, depending on epidemiological, administrative and socioeconomic conditions and systems of government. The success of these programmes depends on suitable education of medical and health personnel, and a flexible approach in guiding antimalarial operations. A major effort to train such personnel is being made by international and national organizations, as an essential prerequisite to the translation of advances in malaria research into useful measures in the field. This clearly and simply written book will without doubt play an important role in such training efforts.

Peter I. Trigg is in the Research and Technical Intelligence Unit, Malaria Action Programme, World Health Organization, Avenue Appia. 1211 Geneva 27, Switzerland.

\section{New editions}

- The Birth of $A$ New Physics: Revised and Updated, by I. Bernard Cohen. Publisher is W.W. Norton, price is $\$ 17.95$. To be published in Britain in October, $£ 15.95$.

- Amino Acid Metabolism, 2nd Edn, by David A. Bender. Publisher is Wiley, price is $\$ 38$, $£ 24$.

- Introduction to Mass Spectrometry, 2nd Edn, by J. Throck Watson. Publisher is Raven, price is $\$ 49$.

- Hematology, 4th Edn, edited by William $S$. Beck. Publisher is MIT Press, price is hbk $\$ 40$, $£ 39.95$; pbk $\$ 18.50, £ 18.50$. 